\title{
High Energy Neutrinos from Blazars
}

\author{
R.J. Protheroe \\ Department of Physics and Mathematical Physics, The University of \\ Adelaide, Adelaide, Australia 5005
}

\begin{abstract}
I describe a model of energetic gamma ray and neutrino emission in blazars which is consistent with most of the gamma-ray observations, and use this model to estimate the diffuse intensity of high energy neutrinos from blazars.
\end{abstract}

\section{Introduction}

The second EGRET catalog of high-energy gamma-ray sources (Thompson 1995) contains 40 high confidence identifications of AGN, and all appear to be blazars. Clearly, the gamma ray emission is associated with the jet. Several of these AGN show gamma-ray variability with time scales of $\sim 1$ day (Kniffen 1993), and this places an important constraint on the models.

Most previous work on gamma ray emission in AGN jets involved electron acceleration and inverse Compton scattering. The proton blazar model was proposed originally by Mannheim and Biermann (1992) and developed by Mannheim (1996). In this model both protons and electrons are accelerated, and protons interact via pion photoproduction and Bethe-Heitler pair production with synchrotron radiation produced by the electrons. Here I consider a model in which protons are accelerated and interact directly with accretion disk radiation.

\section{The model}

If the protons interacted with matter to produce the gamma rays on a timescale consistent with the observed variability, then the proton number density in the blob must be $n_{H}>10^{9} \mathrm{~cm}^{-3}$, assuming a Doppler factor of $\sim 20$. Such a high density would not normally be expected in AGN jets. Interactions with the radiation field are therefore necessary if protons are accelerated, but a sufficiently high density of target photons for proton interactions would present problems for gamma ray escape if the radiation were isotropic. In the present work, the gamma-ray emission region (referred to as "the blob") is placed well above the accretion disk, and any scattering by clouds or coronae is assumed to be negligible, so that the radiation impinging on the blob is highly anisotropic. I use a spectrum which is a modification of the standard thin accretion disk spectrum (Shakura and Sunyaev 1973) in which the maximum temperature is lower than usual to account for the absence of the inner part of the disk due to the presence of the jet. This is only an approximation, and does not include 
the soft $\mathrm{X}$-ray emission. Calculations are in progress using a more appropriate spectrum and will be reported elsewhere. However, the results for GeV gammarays and high energy neutrinos are not expected to differ by very much from those presented here.

From variability arguments, the size of the blob can not be larger than $c \Delta t_{\text {var }}^{\prime}$ (primed quantities refer to the jet frame). This means that the region in which the protons are accelerated and trapped must be smaller than $0.02 \mathrm{pc}$, and proton gyroradii, $r_{g}^{\prime}$, at the highest energies must be less than $0.01 \mathrm{pc}$. The maximum acceleration rate for any acceleration mechanism is $\left(d E^{\prime} / d t^{\prime}\right)_{\max }=$ $e c^{2} B^{\prime}$ where $B^{\prime}$ is the magnetic field in the blob, but for realistic acceleration mechanisms the acceleration rate will be at least a factor of 10 lower. During acceleration, protons will also suffer synchrotron losses and we require that these be less than the energy losses for pion photoproduction at all energies up to the maximum energy (which is determined by pion photoproduction). For a magnetic field of 10 gauss all these conditions are met, giving a maximum jetframe energy of $E_{\max }^{\prime} \approx 1.5 \times 10^{10} \mathrm{GeV}$.

Protons may escape from the blob before interacting, and I assume this is simply due to diffusion to the boundary of the blob where they freely escape. For a reasonable diffusion coefficient, most protons with energies below the threshold for pion photoproduction will escape without interacting.

\section{Interaction of protons and the pair-synchrotron cascade}

We inject an $E^{\prime-2}$ spectrum of protons, continuously randomize their directions in the jet frame, and allow them to interact with radiation. The interactions are treated as described in Szabo and Protheroe (1994) and all pions, and their decay products, are assumed to be produced travelling initially in the direction of the interacting proton.

The gamma rays from $\pi^{0}$ decay may interact with photons from the accretion disk while inside the blob, or outside the blob, or may escape from the AGN. The probability of escaping from the blob and escaping from the AGN are calculated for various angles to the jet axis. The gamma rays that pair produce inside the blob will initiate a pair-synchrotron cascade. The electrons produced inside the blob by photon-photon pair production of gamma-rays from $\pi^{0}$ decay, and electrons from from $\pi^{ \pm}$decay, will synchrotron radiate in the ambient magnetic field and produce the first generation of synchrotron photons in the cascade. I then treat the photon-photon pair production interactions of this first generation of synchrotron gamma-rays in the same way as gamma rays from $\pi^{0}$ decay, the resulting electron positron pairs producing the second generation of synchrotron photons in the cascade. This procedure is repeated for several generations. The resulting gamma ray and neutrino fluxes emerging from the AGN at angles between $2^{\circ}$ and $4^{\circ}$ with respect to the jet axis are shown in Fig. 1.

\section{Neutrino background from unresolved blazars}

I use the luminosity function of gamma-ray loud AGN (Chiang 1995) and integrate over redshift and luminosity to estimate the diffuse background shown in 


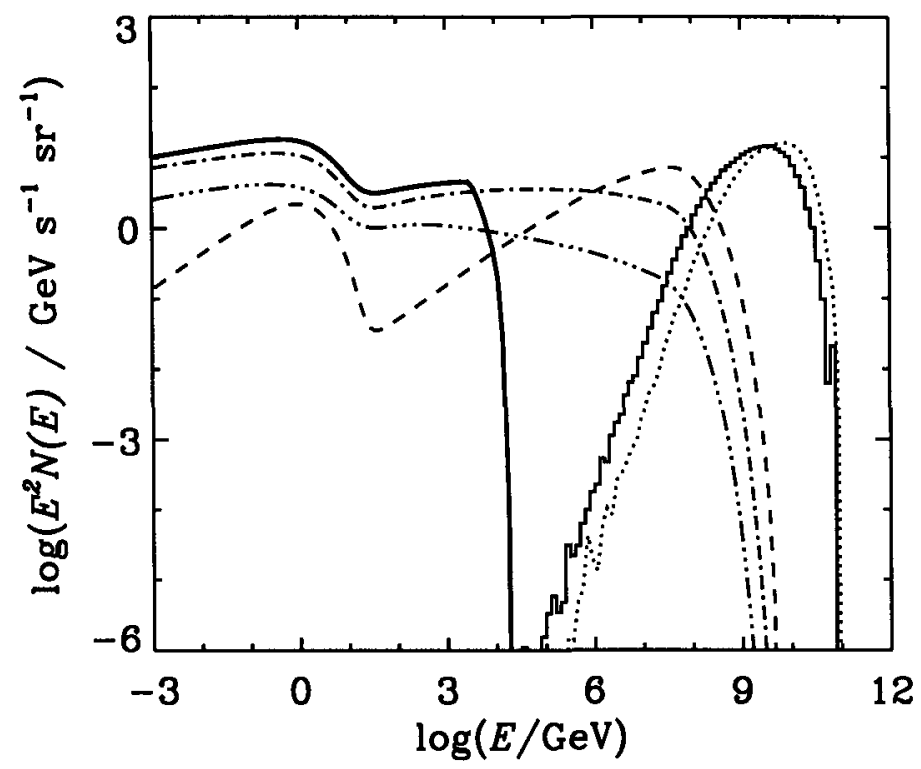

Figure 1. Average gamma ray and neutrino spectra for viewing angles $2^{\circ}-4^{\circ}$ with respect to the jet axis resulting from $p \gamma$ interactions and subsequent cascading. Full hisogram shows the total neutrino output $\left(\nu_{\mu}+\bar{\nu}_{\mu}+\nu_{e}+\bar{\nu}_{e}\right)$, dotted hisogram shows the $\pi^{0}$ gamma spectrum on production, and dashed, dot-dashed, and dot-dot-dot-dashed curves show 1st, 2nd and 3rd generation synchrotron spectra on production. Full curve shows the emerging gamma ray spectrum.

Fig. 2. Also shown is the result of Mannheim (1995) which, given the differences in the models, is in surprisingly good agreement with the present work. The reason for this is that despite the model differences, the most important parameters have similar values (e.g. we inject the same proton spectrum, have similar maximum energies, similar magnetic fields, and similar maximum soft photon energies).

The predicted neutrino intensities may be detectable with the generation of neutrino telescopes currently being constructed (see Gaisser et al. 1995 for a review). For the present work, the number of muon events per year in a detector with an effective collecting area of $2 \times 10^{4} \mathrm{~m}^{2}$ would be $\sim 10$ above $1 \mathrm{TeV}$, and $\sim 6$ above $10 \mathrm{TeV}$, compared with the atmospheric neutrino background of $\sim 225$ and 5 per year respectively (Hill 1996).

As a final note, I mention that one of the EGRET blazars, Markarian 421, which was previously observed at $\mathrm{TeV}$ energies (Punch et al., 1992), very recently had two violent outbursts at $\mathrm{TeV}$ energies with a variability timescale of $\sim 1 \mathrm{~h}$ (Gaidos et al. 1996). Such rapid variability has yet to be adequately explained by any of the models, including that described in the present work. 


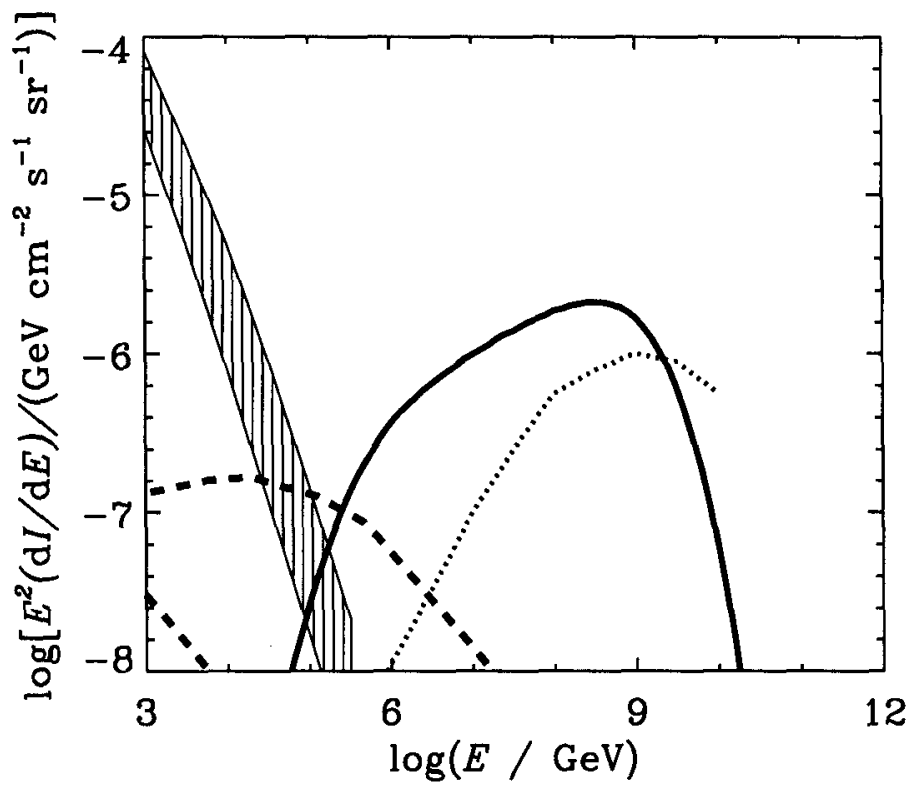

Figure 2. The expected diffuse $\nu_{\mu}+\bar{\nu}_{\mu}$ intensity compared with the atmospheric neutrino intensity (Lipari 1993) (vertical hatched band: the upper curve corresponds to zenith angle $\theta=90^{\circ}$ and the lower curve corresponds to $\theta=0^{\circ}$ ). Also shown are possible minimum and maximum backgrounds due to charm production (dashed lines). Dotted curve shows the result of Mannheim (1995).

\section{References}

Chiang, J., et al., ApJ, 452156 (1995).

Gaidos, J.A., et al., Nature in press (1996).

Gaisser, T.K., Halzen, F., and Stanev, T., Physics Reports, 258, 173 (1995).

Hill, G., Astroparticle Phys. in press (1996).

Kniffen, D.A., et al., ApJ, 411133 (1993).

P. Lipari, Astroparticle Phys. 1195 (1993).

Mannheim, M., and Biermann, P.L., A\&A221, 211 (1989).

Mannheim, K., Astroparticle Phys. 3295 (1995).

Punch, M., et al. Nature 358, 477 (1992).

Shakura, N.I., and Sunyaev, R.A., A\&A, 24337 (1973).

Szabo A. P. and Protheroe R.J. Astroparticle Phys. 2375 (1994).

Thompson, D.J., et al., ApJS, 101259 (1995). 